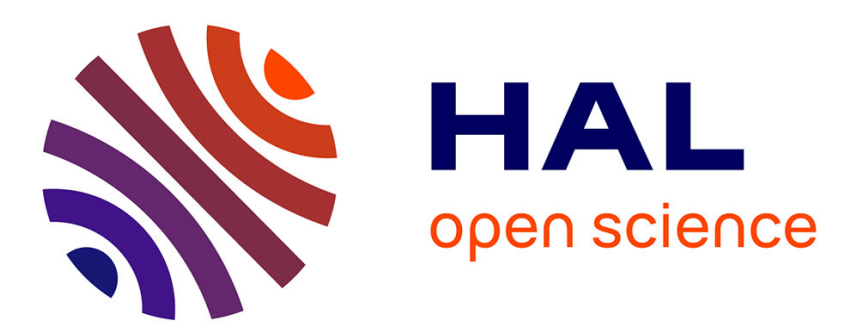

\title{
A Collaborative Environment Perception Approach for Vehicular Ad hoc Networks
}

Sadia Ingrachen, Nadjib Achir, Paul Mühlethaler, Tounsia Djamah, Amine Berqia

\section{- To cite this version:}

Sadia Ingrachen, Nadjib Achir, Paul Mühlethaler, Tounsia Djamah, Amine Berqia. A Collaborative Environment Perception Approach for Vehicular Ad hoc Networks. VTC 2018 - Fall - 2018 IEEE 88th Vehicular Technology Conference, Aug 2018, Chicago, United States. hal-01864608

\section{HAL Id: hal-01864608 \\ https://hal.science/hal-01864608}

Submitted on 30 Aug 2018

HAL is a multi-disciplinary open access archive for the deposit and dissemination of scientific research documents, whether they are published or not. The documents may come from teaching and research institutions in France or abroad, or from public or private research centers.
L'archive ouverte pluridisciplinaire HAL, est destinée au dépôt et à la diffusion de documents scientifiques de niveau recherche, publiés ou non, émanant des établissements d'enseignement et de recherche français ou étrangers, des laboratoires publics ou privés. 


\title{
A Collaborative Environment Perception Approach for Vehicular Ad hoc Networks
}

\author{
Sadia Ingrachen *, Nadjib Achir ${ }^{\dagger}$, Paul Muhlethaler ${ }^{\S}$, Tounsia Djamah*, and Amine Berqia ${ }^{\ddagger}$ \\ *LARI laboratory, Mouloud Mammeri University of Tizi-Ouzou \\ B.P $\mathrm{N}^{\circ} 17 \mathrm{RP}-$ Tizi-Ouzou, Algeria \\ ${ }^{\dagger}$ L2TI Laboratory, Université Paris 13, Sorbonne Paris Cité \\ 99 Avenue J-B Clément, 93430 Villetaneuse, France \\ Email: \{nadjib.achir\}@univ-paris13.fr \\ §INRIA EVA, Centre de Recherche de Paris, 2 Rue Simone, IFF CS 42112, 75589 Paris Cedex 12 \\ Email: \{paul.muhlethaler\}@ inria.fr \\ $\ddagger$ Université Mohammed V de Rabat, ENSIAS, Morocco
}

\begin{abstract}
In this paper, we focus on vehicular safety applications based on the Dedicated Short Range Communication (DSRC) standard. We propose a new mechanism to alleviate channel congestion by reducing the beacons load while maintaining an accurate awareness level. Our scheme is based on the collective perception concept which consists in sharing perceived status information collected by vehicles equipped with different types of sensors (radars, lidars, cameras, etc.). To achieve our goal, we propose two main schemes. The first one consists in implementing the collective perception capability on vehicles and adding a new category of status messages to share locally collected sensor data in order to reduce channels load and enhance vehicles' awareness. The second scheme concerns the accuracy level of the received information from the collective perception enabled vehicles by fixing a prior error threshold on the position. The method proposed is validated by simulations and the results obtained are compared to those of an application based on the traditional beaconing scheme of the IEEE802.11p standard. The simulations show that the proposed scheme is able to significantly reduce the load on the control channel incurred by the beacons and the packet error ratio for different network densities and built-in sensors characteristics.
\end{abstract}

\section{INTRODUCTION}

Nowadays, we are witnessing a tremendous growth in the number of vehicles on roads all over the world. A major consequence of this has been an increase in the number of accidents and inevitably the loss of human life. This is why governments and research communities are investigating the development of passive and active safety solutions for road users. The recent advances in software and hardware technologies and the evolution of wireless communications have assisted researchers and allowed the emergence of a very promising application area of networking. This application concerns Vehicular Ad hoc Networks (VANET). Wireless communications in vehicular environments allow a variety of wide-ranging applications [12] [2]. Before extending their utilization to the infotainment applications, the prime objective of VANETs was to enhance road safety by reducing traffic congestion and accidents. In such VANET applications, vehicles periodically exchange their status information like position, speed, heading, etc. using the IEEE 802.11p [1] [3] standard which is based on on one-hop broadcasts and the 1609 protocol stack. This mechanism allows vehicles to acquire information about traffic conditions in their vicinity. Beaconing is a corner stone component of the V2V communications. However, these periodic beacons lead to the saturation of the channel and packet losses as the IEEE 802.11p MAC layer is based on the Carrier Sense Multiple Access with Collision Avoidance (CSMA/CA) channel access method, which cannot cope with all possible collisions. On the other hand, the vehicular environment is characterized by a rapidly changing the very fluctuating topology and limited radio resources. These characteristics have a negative impact on Quality of Service (QoS), particularly when the network density is high, since a large number of vehicles are simultaneously competing to access the radio channel. In addition, vehicular safety applications have stringent requirements in terms of QoS parameters such as delay and reception rate. Therefore, the most challenging task for cooperative safety communications is how to maintain a satisfactory awareness level while reducing congestion on the radio shared channel. To resolve this issue, different approaches have been explored. The main ones are based on an adaptation of the transmission rate, the transmission power or both. While power adaptation schemes limit the vehicle's neighborhood, and thus the number of vehicles which receive the beacons, rate adaptation schemes try to distribute the bandwidth fairly between the vehicles. In the literature, these tracks have been widely explored in varying network conditions [7] [18] [14] [8] [10] [11] [5] [16]. However, these approaches present drawbacks related to network density and the limited wireless resources.

In this paper we introduce a new beaconing scheme in order to alleviate channel saturation effects on the performance of cooperative safety applications and optimize the network resource utilization. To reduce the exchange of periodic Car Awareness Messages (CAMs), we use the collective perception Concept [6] [4]. Recent technological advances have made possible to design low costs and high performance components for autonomous driving systems where vehicles are equipped with different types of sensors such as Radars, Lidars (Light 
Detection and Ranging), acoustic sensors and cameras. The built-in sensors allow these vehicles to obtain an extended view of their surroundings and share these views via a wireless interface with other vehicles. This asset may be exploited in the network to reduce the number of communications between vehicles as we did in this work.

The rest of the paper is organized as follows. Section II discusses the state of the art of the different approaches proposed to resolve the congestion problem in vehicular environments. Section III deals with our mechanism to resolve the channel load problem. In Section IV, simulations are conducted to evaluate the performance of the mechanism proposed and its efficiency. In section $\mathrm{V}$ the simulation results are discussed. Finally, Section VI concludes the paper.

\section{RELATED WORKS}

The beaconing adaptation issue has been widely studied and several approaches have been proposed to cope with the overload and saturation of $\mathrm{CCH}$ in $\mathrm{V} 2 \mathrm{~V}$ communications. These approaches usually rely on the aforementioned schemes.

The authors in [14] present a new mechanism based on the adaptation of transmission power and contention window size to improve the efficiency of information dissemination. Transmission power adaptation relies on estimating the local density of vehicles, whereas the contention window size is adapted to the collision rate.

In [8] the authors propose a Bi-beacon level scheme where two types of beacons are emitted, while keeping the same transmission rate. The first type of beacons with a long transmission range to guarantee large awareness in the network and the second one with a short transmission range to give drivers time to react efficiently.

In [18], the authors propose a combined rate/power adaptation scheme to deal with the channel load in order to improve channel utilization and reduce collisions. The method uses the current collision rate to adjust the transmission parameters. By using a binary search algorithm, the optimal channel load is located and then used by vehicles. This approach results in a reduced the beacon rate while maintaining an acceptable level of awareness for closer neighbors.

A Cooperative Safety Zone is proposed in [7]. The basic idea of the protocol consists in selecting the minimum transmission power for CAMs to keep vehicles connected within a safety zone. The transmission power is selected based on the distance between a source vehicle and its critical neighbors. Cooperative piggybacking is used to extend awareness beyond the safety zone.

Another approach, where the vehicle's driving context is considered, is studied in [16]. To reduce channel loads and give better network performance, the transmission parameters are adjusted according to the application's security requirements and driving conditions.

In [10], power adjustment is made dynamically according to channel status, the history of transmission delays and the message priority. The method is able to achieve better performance than the DSRC standard where the transmission power is fixed in advance.

In order to provide high reliability to disseminated beacons, [11] proposes a solution where the beacon adaptation rate is based on the channel load evaluated by two parameters which are the channel busy ratio (CBR) and the local density observed by the vehicles.

In [5], the authors use the concept of collective perception to achieve a decentralized congestion control where different sensing capacities of the vehicles are exploited to enhance traffic awareness and alleviate channel congestion. This approach is efficient when used to reduce channel congestion.

In this paper, we address the channel load control from a different angle. Instead of making use of power/rate adaptation mechanisms like in the previously presented approaches, which are closely related to network dynamics, we will take advantage of different sensing technologies integrated in recent vehicles, which is a promising avenue for the automotive industry and autonomous vehicles.

\section{PROposed MECHANISM}

In this paper, we propose a new mechanism to enhance QoS of cooperative safety communications in vehicular environments and mitigate the channel load issue introduced above. To meet this requirement, our idea consists in using collective perception. The collective perception concept in intelligent transportation systems involves sharing gathered and preprocessed sensor data about the local view of traffic dynamics between connected vehicles. The cooperation between vehicles is carried out via cooperative wireless communication technologies such as V2X (vehicle to everything) communication [13]. In this way, all equipped vehicles participate in extending the awareness information to others beyond their Field of View (FoV). The sensor data fusion models are beyond the scope of this paper.

In the solution given in this paper, we assume that all vehicles are equipped with GPS that provide them with information about their positions and driving status and DSRC transceivers to exchange their own information via wireless messages. In addition to this and unlike existing studies, a second category of vehicles is assumed to be equipped with different types of sensors (cameras, acoustic sensors, radars, lidars ..etc) allowing them to have a vision of the surrounding vehicles. The latter category has an important role in the network as it contributes greatly to reducing channel congestion as will be demonstrated by simulations later. Additionally, we suppose that no obstacles exist on the road and that the perceived dynamic objects consist only of vehicles. A vehicle is described by a unique identifier and its dynamic measurements such as position, speed, heading, etc.

Figure 1 illustrates how our method works. Vehicles A and $\mathbf{B}$ are collective perception enabled. Collected and fused information generated by the different sensors of vehicle $\mathbf{A}$ and $\mathbf{B}$ is included in an Extended CAM (ECAM) where each perceived vehicle is described by its unique identifier, position, heading and velocity. The ECAM contains the description of 


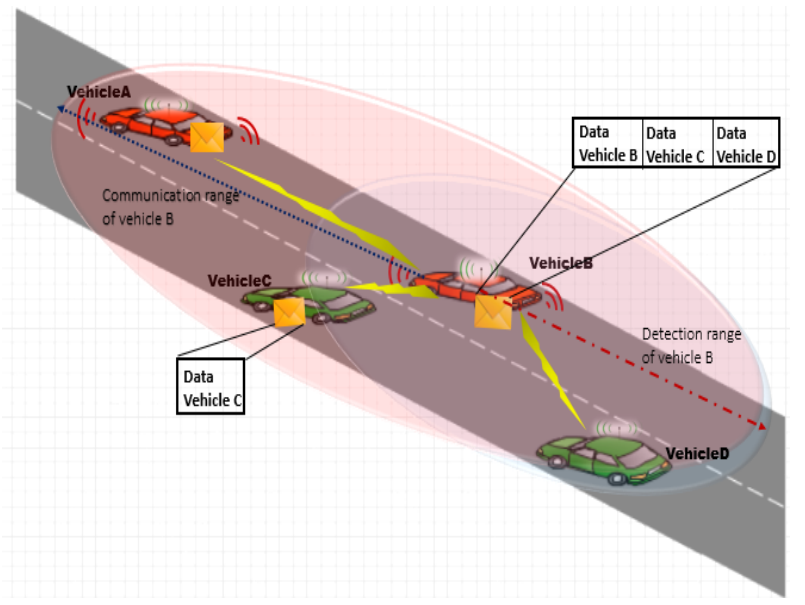

Figure 1: Detection of vehicles in the FoV and sharing their information with vehicles in the communication range

the origin vehicle in a similar way to the CAM. In addition to the self-assessment of the dynamic status of the origin vehicle $\mathbf{B}$, similar descriptors are added for the perceived vehicles $\mathbf{C}$ and $\mathbf{D}$. These messages are shared with all vehicles in the communication range of vehicle $\mathrm{B}$ and allow an extended view of the network. For instance, vehicle $\mathbf{A}$ will be aware of the position of vehicle $\mathbf{D}$ even if it is not within its detection or communication range. On the other hand, when the vehicles $\mathbf{C}$ and $\mathbf{D}$ receive the ECAM from $\mathrm{B}$, they verify the exactitude of the estimation of $\mathrm{B}$ about their own information. If the sensitivity threshold is respected, $\mathbf{C}$ and $\mathbf{B}$ will not send the beacons containing only their information since all the vehicles in the vicinity are aware of their correct position. Otherwise, the vehicles disseminate their beacons.

Before the descriptor of a vehicle is added to the ECAM, the distance between the origin vehicle and the perceived vehicle is calculated in order to ensure that only vehicles in the communication range of the vehicle are included in its neighborhood table. Since sensors perceived information is error-prone, its data precision may be altered as distance increases or due to different intern or environmental factors. So, in order to make our model more realistic, the estimation errors are previewed. In this paper, we consider an error rate in the longitudinal position while estimating neighbors' positions.

In our method, the transmission will be triggered by crossing an error threshold on the vehicles' positions. The decision of a vehicle to disseminate or not its own information at any time depends on the information received from the equipped vehicles. The dynamic information included in the objects descriptors represent what all the vehicles in the communication range of the origin vehicle consider it to be. If the estimation made by the equipped vehicles is not exact, the receiving vehicle broadcasts its status information. Otherwise, there is no need to send the beacons since vehicles in the vicinity are aware of its positions thanks to the ECAM sent by the equipped vehicles.

The vehicles' movement is taken into consideration while choosing the error threshold. For instance, when an equipped vehicle $V_{i}$ sends, at instant $t$, an ECAM containing the estimation of the position of $V_{j} \tilde{P}_{i j}(t)$, the position of the receiving vehicle $V_{j}$ is $\widehat{P_{j}}(t)$, where $\widehat{P}_{j}(t+1)=\tilde{P}_{i j}(t)+\varepsilon_{l o n g}$ with $\varepsilon_{\text {long }}$ being the error induced by the vehicle movement on the longitudinal axis. When a vehicle is moving fast, the error threshold may be frequently crossed. In such a situation, messages will be sent frequently. On the other hand, when the vehicle's acceleration is lower, the position estimation is more accurate, the error threshold is respected and hence messages will be broadcast less often.

\section{Performance Evaluation}

In this section, we evaluate our mechanism with different parameters to investigate the impact of the vehicle density, sensors sensitivity and percentage of equipped vehicles on the performance of the safety applications in terms of packet error rate, channel busy ratio, latency represented by the number of backoffs performed by a vehicle before sending it packet in addition to the beacons load. We conducted several simulations using the simulation framework for vehicular networks Veins [17] and the microscopic traffic simulator Simulation of Urban Mobility [9]. The road scenario we used is a bidirectional $5 \mathrm{~km}$ three-lane section. The simulation parameters are summarized in Table 1.

Table I: Simulation parameters

\begin{tabular}{|c||c|}
\hline Simulation parameter & Value \\
\hline Simulation time & $400 \mathrm{~s}$ \\
Packet size & 32 bytes \\
ECAM size & 202 bytes \\
bit rate & $3 \mathrm{Mbps}$ \\
beacon interval & $20 \mathrm{~ms}$ \\
max vehicles speed & $70 \mathrm{~km} / \mathrm{h}$ \\
Number of Nodes & $(50,100, \ldots, 200) \mathrm{veh} / \mathrm{km}$ \\
Number of Equipped Nodes & $(5,10,20,50,100) \%$ \\
Highway length & $5 \mathrm{~km}(3 \mathrm{lanes})$ \\
Number of runs & 30 \\
Error Threshold & $1.0 \mathrm{~m}$ \\
Detection range & $40 \mathrm{~m}$ \\
Confidence interval & $95 \%$ \\
\hline
\end{tabular}

The results are compared with a simple application based on the basic IEEE 802.11p standard. The metrics we used to evaluate our mechanism are the beacons load which consists of the number of packets sent by each vehicle during the simulation time. The second metric is the packet error rate which represents the packet loss rate due to bit error (SINR not achieved). The channel busy ratio (CBR) is also considered, which represents the ratio of the total time that a vehicle occupies the channel while transmitting divided by the simulation time:

$$
C B R_{i}=\text { total_busy_time }{ }_{i} / \text { sim_time }
$$

Where $C B R_{i}$ is the average CBR that a given vehicle $i$ observes throughout all the simulation time. The last one is the total number of backoff intervals a vehicle waits before sending it packets. In order to show the effectiveness of our 
scheme, extensive simulation was conducted with different market penetration rates.

Simulation results presented in this section evaluate our method for different parameters along with an application based on the standard periodic beaconing scheme proposed in DSRC. According to the following simulation results, we can attest that the impact of our mechanism based on the dissemination of sensors collected information is noteworthy.

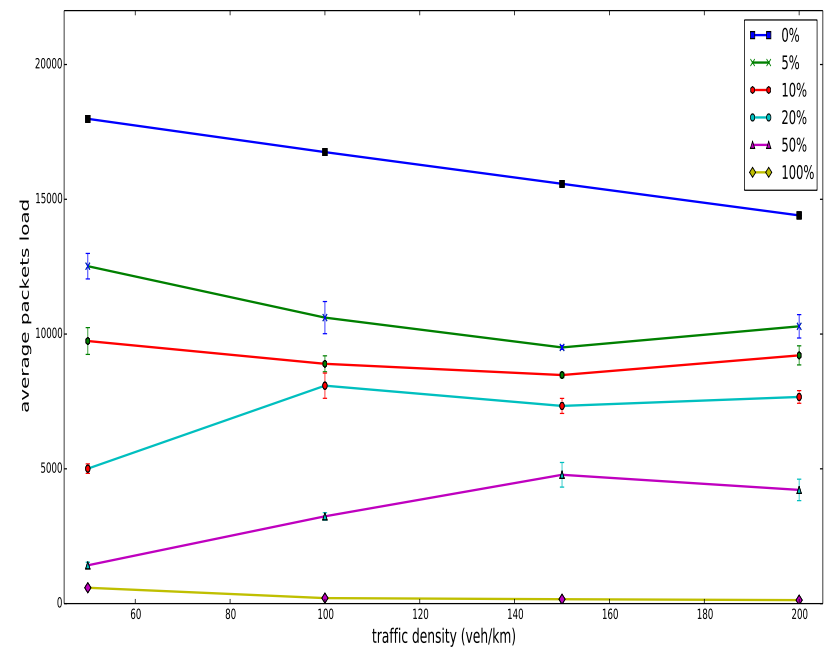

Figure 2: Average packet load versus vehicle density, $T h r=$ $1 \mathrm{~m}, \operatorname{det}_{r}=40 \mathrm{~m}$

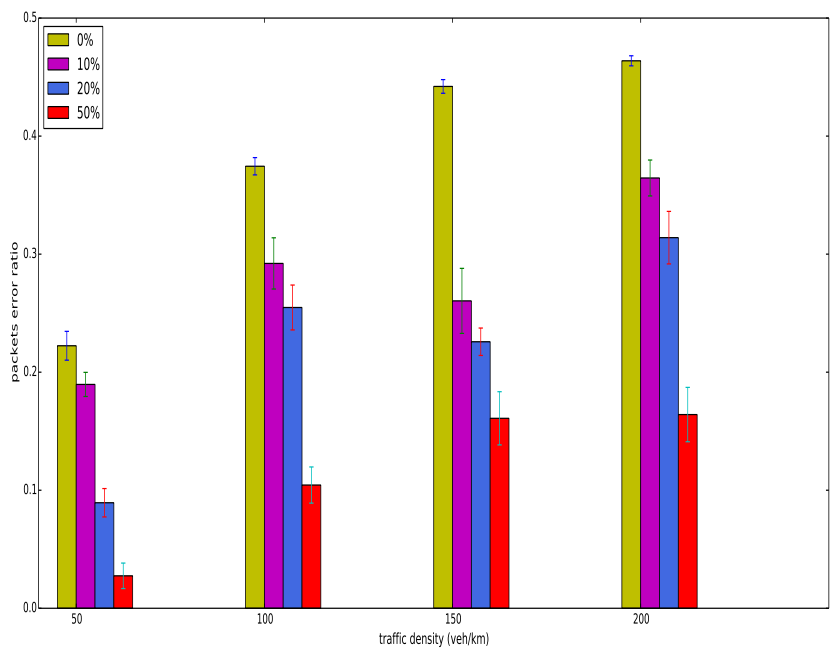

Figure 3: Packet error ratio versus traffic density, $T h r=1 \mathrm{~m}$, $\operatorname{det}_{r}=40 \mathrm{~m}$

Figure 2 depicts the average packet load for different percentages of vehicles with collective perception capabilities with different network densities. We can see that the packet load decreases as the market penetration rate increases. For instance, for $100 \%$ and $20 \%$ penetration rates of the collective perception, the packet load is reduced by $98 \%$ and $46 \%$ respectively without degrading the vehicles' awareness with a $40 \mathrm{~m}$ sensors detection range and an error threshold on the vehicle's longitudinal position of $1 \mathrm{~m}$. With high densities, we see that the proposed mechanism achieves better performance than periodic beaconing.

In Figure 3 the packet error ratio due to a bit error is represented and compared to an application where the standard beaconing scheme of IEEE 802.11p is used. It shows the advantage of using a collective perception-based beaconing scheme compared to the standard periodic beaconing. We can note that our solution alleviates packet losses in the network compared to standard solutions. For $20 \%$ and $50 \%$ we attest that the loss rate is significantly reduced since the packet load is very low. For example, with $100 \%$, the PER is almost zero because the packet load is very low with an improvement of 97\% compared to the IEEE 802.11 p fixed rate beacons.

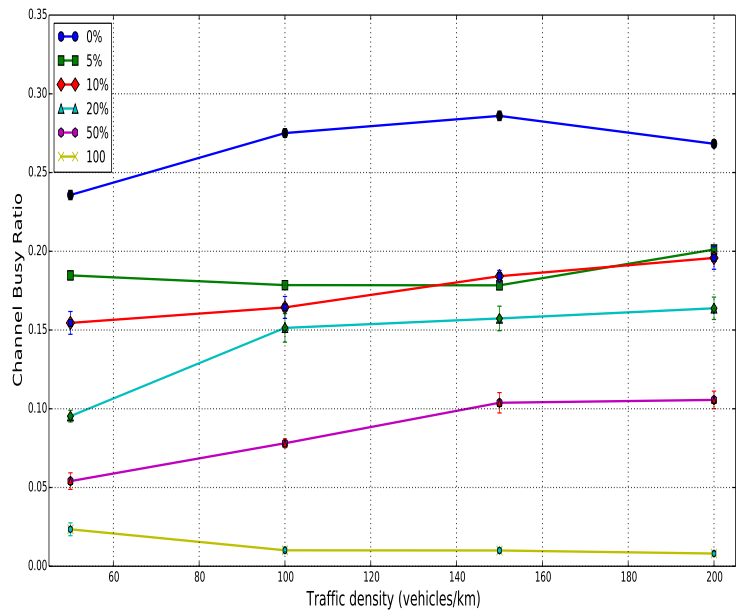

Figure 4: Channel busy ratio versus vehicle density, $T h r=1 \mathrm{~m}$, $\operatorname{det}_{r}=40 \mathrm{~m}$

CBR represents the amount of time that a transmission at the level of the MAC layer of a vehicle is not feasible because the channel is busy. A high value of the CBR reflects a high congestion level of the wireless channel. This is why CBR is considered as the best indicator of the performance in the IEEE 802.11p MAC layer [15]. Figure 4 shows the CBR for different rates of equipped vehicles with different network densities. We can see that in case of $0 \%$, the standard beaconing may cause high channel congestion since all vehicles compete to send their CAMs periodically. Channel congestion becomes worse when traffic density increases. For $20 \%$ and $50 \%$, the CBR is significantly reduced, as is the packet loss rate, as depicted in Figure 3.

As collective perception extends the awareness range of vehicles by sharing sensor data in additional cooperative messages, it significantly reduces the packets overhead, and hence channel congestion while reducing packet losses and delays. 


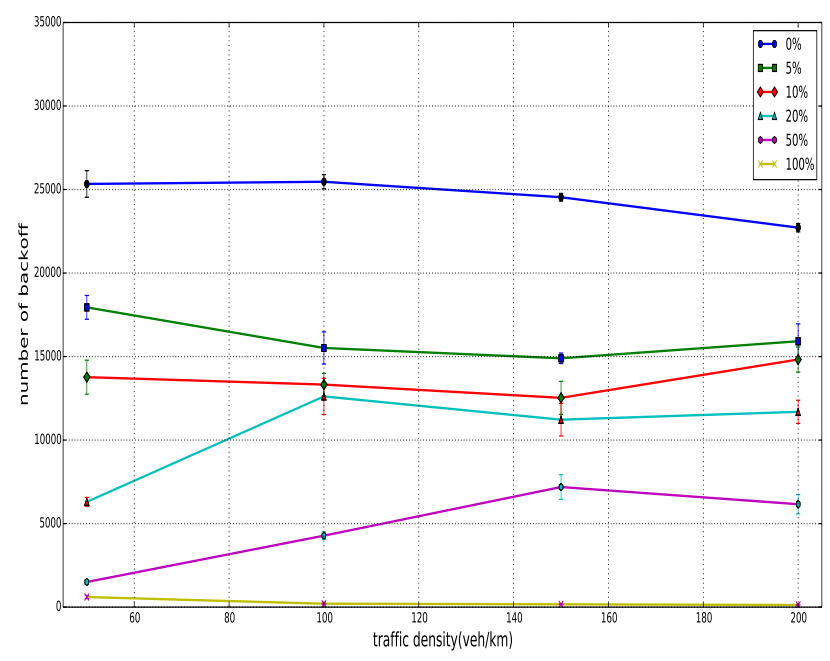

Figure 5: Number of backoffs for different rates of equipped vehicles at different densities, $T h r=1 \mathrm{~m}, \operatorname{det}_{r}=40 \mathrm{~m}$

Figure 5 depicts the important improvements of the packets' latency, expressed by the number of backoff slots performed by a node, achieved by our method in comparison to the standard periodic beaconing scheme. We can notice that the collective perception mechanism allows a significant reduction in the packets access delays. This is mainly due to the decrease in the packet load, hence the decrease of contention to access the channel. In this case, a node wishing to disseminate its beacons will not perform many backoff intervals before sensing the channel free. Even with of high densities, an important gain can be seen. For example, in $200 \mathrm{veh} / \mathrm{km}$ traffic density, a gain of $28 \%$ and $72 \%$ is achieved by a $5 \%$ and $50 \%$ of equipped vehicles respectively compared to $0 \%$.

\section{CONCLUSION}

In this paper, we have presented a new collective perception mechanism to reduce the channel congestion issue due to CAMs in vehicular environments and enhance the QoS of cooperative safety applications in terms of the packet load, PER and average channel busy time. The vehicles with collective perception capacity share the collected information from the built-in sensors with other vehicles. We introduce a new type of message to share sensor data that we call ECAM. After the reception, vehicles verify the accuracy of the information included in the message. According to the result, a transmission could be triggered or not as required by the mechanism. The simulations conducted show that our scheme performs well under different network conditions such as variable traffic densities and percentage of vehicles equipped with our mechanism compared to the periodic beaconing scheme proposed by the DSRC standard.

\section{REFERENCES}

[1] IEEE Standards Association et al. Draft standard for wireless access in vehicular environments (wave)-multi-channel operation. IEEE Std 1609.4-2010, 2010
[2] A. Bujari, O. Gaggi, C. E. Palazzi, and D. Ronzani. Would current ad-hoc routing protocols be adequate for the internet of vehicles? a comparative study. IEEE Internet of Things Journal, pages 1-1, 2018.

[3] IEEE 802.11 Working Group et al. Part11: Wireless lan medium access control (mac) and physical layer (phy) specifications. ANSI/IEEE Std. 802.11, 1999.

[4] Hendrik-Jörn Günther, Björn Mennenga, Oliver Trauer, Raphael Riebl, and Lars Wolf. Realizing collective perception in a vehicle. In Vehicular Networking Conference (VNC), 2016 IEEE, pages 1-8. IEEE, 2016.

[5] Hendrik-Jorn Günther, Raphael Riebl, Lars Wolf, and Christian Facchi. Collective perception and decentralized congestion control in vehicular ad-hoc networks. In Vehicular Networking Conference (VNC), 2016 IEEE, pages 1-8. IEEE, 2016.

[6] Hendrik-jorn Gunther, Oliver Trauer, and Lars Wolf. The potential of collective perception in vehicular ad-hoc networks. In ITS Telecommunications (ITST), 2015 14th International Conference on, pages 1-5. IEEE, 2015.

[7] Muhammad A Javed and Jamil Y Khan. A cooperative safety zone approach to enhance the performance of vanet applications. In Vehicular Technology Conference (VTC Spring), 2013 IEEE 77th, pages 1-5. IEEE, 2013.

[8] Bongkyu Kim, Heecheol Song, and Hwang Soo Lee. Bi-beacon: Twolevel power control for enhancing beacon performance in vehicular ad hoc networks. In Connected Vehicles and Expo (ICCVE), 2014 International Conference on, pages 1000-1001. IEEE, 2014.

[9] Daniel Krajzewicz, Jakob Erdmann, Michael Behrisch, and Laura Bieker. Recent development and applications of sumo-simulation of urban mobility. International Journal On Advances in Systems and Measurements, 5(3\&4):128-138, 2012.

[10] Guofeng Lei, Fuqiang Liu, Ping Wang, Xinhong Wang, and Ning Wang. Power adjustment based congestion control in vehicular ad-hoc networks. In Artificial Intelligence with Applications in Engineering and Technology (ICAIET), 2014 4th International Conference on, pages 280-285. IEEE, 2014.

[11] Linying Liu, Xinhong Wang, Chao Wang, and Fuqiang Liu. An efficient beacon rate adaptation scheme for vehicular networks. In Wireless Communications \& Signal Processing (WCSP), 2013 International Conference on, pages 1-6. IEEE, 2013.

[12] C. E. Palazzi, A. Bujari, G. Marfia, and M. Roccetti. An overview of opportunistic ad hoc communication in urban scenarios. In 2014 13th Annual Mediterranean Ad Hoc Networking Workshop (MED-HOCNET), pages 146-149, June 2014.

[13] Andreas Rauch, Felix Klanner, and Klaus Dietmayer. Analysis of v2x communication parameters for the development of a fusion architecture for cooperative perception systems. In Intelligent Vehicles Symposium (IV), 2011 IEEE, pages 685-690. IEEE, 2011.

[14] Danda B Rawat, Dimitrie C Popescu, Gongjun Yan, and Stephan Olariu. Enhancing vanet performance by joint adaptation of transmission power and contention window size. IEEE Transactions on Parallel and Distributed Systems, 22(9):1528-1535, 2011.

[15] Ali Rostami, Bin Cheng, Gaurav Bansal, Katrin Sjöberg, Marco Gruteser, and John B Kenney. Stability challenges and enhancements for vehicular channel congestion control approaches. IEEE Transactions on Intelligent Transportation Systems, 17(10):2935-2948, 2016.

[16] Miguel Sepulcre, Javier Gozalvez, Jerome Harri, and Hannes Hartenstein. Application-based congestion control policy for the communication channel in vanets. IEEE Communications Letters, 14(10):951-953, 2010.

[17] Christoph Sommer, Reinhard German, and Falko Dressler. Bidirectionally coupled network and road traffic simulation for improved ivc analysis. IEEE Transactions on Mobile Computing, 10(1):3-15, 2011.

[18] Sofiane Zemouri, Soufiene Djahel, and John Murphy. Smart adaptation of beacons transmission rate and power for enhanced vehicular awareness in vanets. In Intelligent Transportation Systems (ITSC), 2014 IEEE 17th International Conference on, pages 739-746. IEEE, 2014. 